\title{
A Novel MIMO - OFDM Technique for Improving Wireless Communications System Performance based on SF-BC
}

\author{
Ali Jasim Ramadhan \\ Computer Engg. Tech. \\ Univ. College of Humanity Studies \\ Najaf, Iraq
}

\begin{abstract}
In this work, the author implemented a NOVEL technique of multiple input multiple output (MIMO) orthogonal frequency division multiplexing (OFDM) based on space frequency block coding (SF-BC). Where, the implemented code is designed based on the QOC using the techniques of the reconfigurable antennas. The proposed system is implemented using MATLAB program, and the results showing best performance of a wireless communications system of higher coding gain and diversity.
\end{abstract}

\section{Keywords}

MIMO, OFDM, SF-BC, QOC.

\section{INTRODUCTION}

Orthogonal frequency division multiplexing (OFDM) is a modulation of multi carrier technique which has high spectral efficiency and higher data rates [1].

In OFDM, the fading channel of the frequency selective is transformed into several sub flat fading channels by the division of the channel bandwidth [2]. OFDM can be combined with MIMO to increase the capacity of the system and performance [3].

The capacity and reliability of wireless communications system can be increase by using the reconfigurable antennas too [4]

Therefor, the performance of the wireless systems can improve by using the reconfigurable antennas and designing a proper code [5].

In order to access the advantages of this technique, literature review are done and the efficient code must be design.

In [6], designed a MIMO based on the reconfigurable receiver antenna which achieved a diversity that equals to the product of the number of receive and transmit antennas, and the number of reconfigurable states of the receive antennas. Where, their gain diversity is possible only under the propagation that selected in the channel.

In [7], designed the system based on the reconfigurable transmitter and receiver antennas. Where, they could not achieve the diversity of the frequency between $T_{x}$ and $R_{x}$.

In [8], used the space time coding to get the first frequency diversity in multipath environment.

In [9]-[10], they designed similar SF in MIMO OFDM system.

So, the SF codes that obtained can not achieved spatial and frequency diversities togethers.
Therefor in [11], they used a subcarrier technique to improve the diversity gain and minimize the complexity in the receiver side.

In [12], they used the mapping technique to exploit the full frequency but it does not ensure the full code.

$\mathrm{BC}$ that obtained the frequency diversity was designed [13], [14] and [5].

So, in the above works and many other similar works, the SF codes that proposed can not be able to achieve the full diversity in the MIMO OFDM reconfigurable antenna techniques.

\section{TECHINQUE DESCRIPTION}

In this work, the author implemented a coding to improve the performance of the MIMO - OFDM system.

His method include a code that is sent over $T_{x}$ antennas, OFDM tones, and radiation states. In order to obtain state diversity, he configured each transmit antenna element to independently switch its radiation pattern to a direction that can be selected using criteria, e.g., to reduce the correlation of radiation states. The implemented code is based on the quasi orthogonal space time block codes (QOSTBC) [15]-[16].

This paper is prepared as follows. In Section 3, introduce the system model. In Section 4, discuss the SF - BC design. In Section 5, view the results. In Section 6, conclusions are presente.

\section{SYSYTEM MODEL}

Assume that the "MIMO-OFDM" proposed system is shown in Fig. 1.

In this system, the author assumed that the omni-directional antenna of receiver " $\mathrm{M}_{\mathrm{r} \text { " }}$ is fixed radiation, and the OFDM modulation " $\mathrm{N}_{\mathrm{c} \text { " }}$ and FSF channels with propagation paths " $\mathrm{L}$ " between each pair of $\mathrm{M}_{\mathrm{t}}$ and $\mathrm{M}_{\mathrm{r}}$ in each the state of radiation.

The channel IR between $M_{t} i$ and receive $M_{r} j$ in the $p$-th radiation state is:

$$
h_{p}^{i, j}(\tau)=\sum_{l=0}^{L-1} \alpha_{p}^{i, j}(l) \delta\left(\tau-\tau_{l, p}\right),
$$




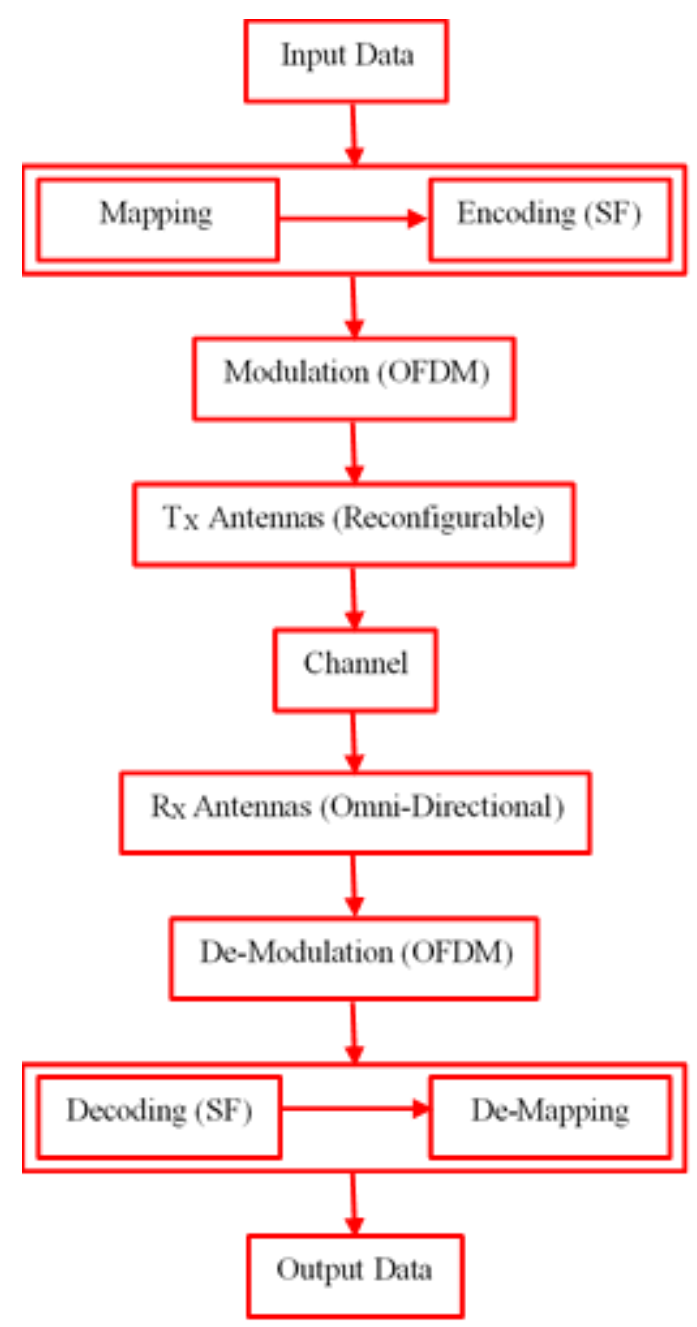

Fig.1 Block Diagram of the Proposed System.

The FR of the channel at the $n$-th subcarrier between $M_{t} i$ and $\mathrm{M}_{\mathrm{r}} \mathrm{j}$ is:

$$
H_{p}^{i, j}(n)=\sum_{l=0}^{L-1} \alpha_{p}^{i, j}(l) e^{-j 2 \pi n \Delta f \tau_{l, p}}
$$

The transmitted SF code, $\mathrm{C}_{\mathrm{p}} \in \mathrm{CMt}^{\mathrm{XNc}}$, is:

$$
\mathbf{C}_{p}=\left[\begin{array}{cccc}
c_{p}^{1}(0) & c_{p}^{1}(1) & \cdots & c_{p}^{1}\left(N_{c}-1\right) \\
c_{p}^{2}(0) & c_{p}^{2}(1) & \cdots & c_{p}^{2}\left(N_{c}-1\right) \\
\vdots & \vdots & \ddots & \vdots \\
c_{p}^{M_{t}}(0) & c_{p}^{M_{t}}(1) & \cdots & c_{p}^{M_{t}}\left(N_{c}-1\right)
\end{array}\right]
$$

At the receiver, the received subcarrier and radiation signal can be written as:

$$
y_{p}^{j}(n)=\sqrt{\frac{\gamma}{M_{t}}} \sum_{i=1}^{M_{t}} H_{p}^{i, j}(n) c_{p}^{i}(n)+z_{p}^{j}(n),
$$

The received signal is:

$$
\mathbf{y}_{p}=\sqrt{\frac{\gamma}{M_{t}}} \mathbf{H}_{p} \mathbf{c}_{p}+\mathbf{z}_{p}
$$

Where:

$$
\mathbf{H}_{p}=\operatorname{diag}\left\{\mathbf{H}_{p}(0), \mathbf{H}_{p}(1), \cdots, \mathbf{H}_{p}\left(N_{c}-1\right)\right\}
$$

The $\mathrm{P}$ radiation states $\mathrm{SF}$ code is:

$$
\mathbf{C}=\left[\mathbf{C}_{1}, \mathbf{C}_{2}, \cdots, \mathbf{C}_{P}\right] \text {, }
$$

The over all radiation states of the received signals is:

$$
\mathbf{y}=\sqrt{\frac{\gamma}{M_{t}}} \mathbf{H} \mathbf{c}+\mathbf{z}
$$

Where: $\mathrm{c}$ is the channel matrix, and $\mathrm{z}$ is the noise vector.

\section{SF - BC DESIGN}

In this section, the author present his proposed code. $\mathrm{He}$ designed the code based on the QOC. Where, the SF code is:

$$
\mathbf{C}_{p}=\left[\mathbf{G}_{p}^{1^{T}} \mathbf{G}_{p}^{2^{T}} \cdots \mathbf{G}_{p}^{M^{T}} \mathbf{0}_{N_{c}-M L M_{t}}^{T}\right]
$$

Where:

$$
\mathbf{G}_{p}^{m}=\operatorname{col}\left\{\mathbf{X}_{1}, \mathbf{X}_{2}, \cdots, \mathbf{X}_{L}\right\}
$$

Assume having $\mathrm{M}_{\mathrm{t}}=2$, so:

$$
\mathbf{A}\left(x_{1}, x_{2}\right)=\left[\begin{array}{cc}
x_{1} & x_{2} \\
-x_{2}^{*} & x_{1}^{*}
\end{array}\right],
$$

So:

$$
\mathbf{G}_{p}^{m}=\left[\begin{array}{c}
\mathbf{A}\left(\mathcal{S}_{2(p-1) L+1}^{m}, \mathcal{S}_{2(p-1) L+2}^{m}\right) \\
\mathbf{A}\left(\mathcal{S}_{2(p-1) L+3}^{m}, \mathcal{S}_{2(p-1) L+4}^{m}\right) \\
\vdots \\
\mathbf{A}\left(\mathcal{S}_{2 p L-1}^{m}, \mathcal{S}_{2 p L}^{m}\right)
\end{array}\right]
$$

In (12), can compute as:

$$
\begin{aligned}
& {\left[\begin{array}{lll}
\mathcal{S}_{1}^{m} \mathcal{S}_{3}^{m} \cdots & \mathcal{S}_{2 P L-1}^{m}
\end{array}\right]^{T}=\Theta\left[\begin{array}{llll}
s_{1}^{m} & s_{3}^{m} & \cdots & s_{2 P L-1}^{m}
\end{array}\right]^{T},} \\
& {\left[\begin{array}{lllll}
\mathcal{S}_{2}^{m} \mathcal{S}_{4}^{m} & \cdots & \mathcal{S}_{2 P L}^{m}
\end{array}\right]^{T}=\Theta\left[\begin{array}{llll}
s_{2}^{m} & s_{4}^{m} & \cdots & s_{2 P L}^{m}
\end{array}\right]^{T},}
\end{aligned}
$$

There are many optimization methods can be used to find the objective function, such as: "the minimum Euclidean distance betIen constellation points".

For example, assume that the proposed system with these properties: $\mathrm{M}_{\mathrm{t}}=2, \mathrm{P}=2$, and $\mathrm{L}=2$. Therefor, the $\mathrm{C}_{1}$ and $\mathrm{C}_{2}$ are:

$$
\begin{aligned}
\mathbf{C}_{1} & =\frac{1}{4}\left[\begin{array}{ccccccccc}
\mathcal{S}_{1}^{1} & -\mathcal{S}_{2}^{1^{*}} & \mathcal{S}_{3}^{1} & -\mathcal{S}_{4}^{1^{*}} & \ldots & \mathcal{S}_{1}^{M} & -\mathcal{S}_{2}^{M^{*}} & \mathcal{S}_{3}^{M} & -\mathcal{S}_{4}^{M^{*}} \\
\mathcal{S}_{2}^{1} & \mathcal{S}_{1}^{1 *} & \mathcal{S}_{4}^{1} & \mathcal{S}_{3}^{1 *} & \ldots & \mathcal{S}_{2}^{M} & \mathcal{S}_{1}^{M *} & \mathcal{S}_{4}^{M} & \mathcal{S}_{3}^{M *}
\end{array}\right] \\
\mathbf{C}_{2} & =\frac{1}{4}\left[\begin{array}{ccccccccc}
\mathcal{S}_{5}^{1} & -\mathcal{S}_{6}^{1^{*}} & \mathcal{S}_{7}^{1} & -\mathcal{S}_{8}^{1^{*}} & \ldots & \mathcal{S}_{5}^{M} & -\mathcal{S}_{6}^{M^{*}} & \mathcal{S}_{7}^{M} & -\mathcal{S}_{8}^{M^{*}} \\
\mathcal{S}_{6}^{1} & \mathcal{S}_{5}^{1 *} & \mathcal{S}_{8}^{1} & \mathcal{S}_{7}^{1 *} & \ldots & \mathcal{S}_{6}^{M} & \mathcal{S}_{5}^{M *} & \mathcal{S}_{8}^{M} & \mathcal{S}_{7}^{M *}
\end{array}\right]
\end{aligned}
$$

The entries of $\mathrm{C}_{\mathrm{p}}$ are computed using (13). As a result, obtain $\mathrm{C}_{1}^{\mathrm{T}}$ as: 


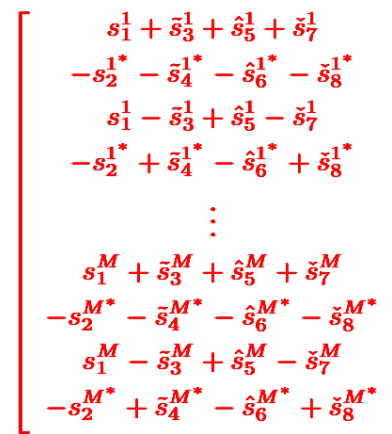

$$
\left.\begin{array}{c}
s_{2}^{1}+\tilde{s}_{4}^{1}+\hat{s}_{6}^{1}+\check{s}_{8}^{1} \\
s_{1}^{1^{*}}+\tilde{s}_{3}^{1^{*}}+\hat{s}_{5}^{1^{*}}+\check{s}_{7}^{1^{*}} \\
s_{2}^{1}-\tilde{s}_{4}^{1}+\hat{s}_{6}^{1}-\check{s}_{8}^{1} \\
s_{1}^{1^{*}}-\tilde{s}_{3}^{1^{*}}+\hat{s}_{5}^{1^{*}}-\check{s}_{7}^{1^{*}} \\
\vdots \\
s_{2}^{M}+\tilde{s}_{4}^{M}+\hat{s}_{6}^{M}+\check{s}_{8}^{M} \\
s_{1}^{M^{*}}+\tilde{s}_{3}^{M^{*}}+\hat{s}_{5}^{M^{*}}+\check{s}_{7}^{M^{*}} \\
s_{2}^{M}-\tilde{s}_{4}^{M}+\hat{s}_{6}^{M}-\check{s}_{8}^{M} \\
s_{1}^{M^{*}}-\tilde{s}_{3}^{M^{*}}+\hat{s}_{5}^{M^{*}}-\check{s}_{7}^{M^{*}}
\end{array}\right]
$$

And $\mathrm{C}_{2}{ }^{\mathrm{T}}$ as:

$$
\left[\begin{array}{cc}
s_{1}^{1}+\bar{s}_{3}^{1}-\hat{s}_{5}^{1}-\check{s}_{7}^{1} & s_{2}^{1}+\bar{s}_{4}^{1}-\hat{s}_{6}^{1}-\check{s}_{8}^{1} \\
-s_{2}^{1^{*}}-\tilde{s}_{4}^{1^{*}}+\hat{s}_{6}^{1^{*}}+\check{s}_{8}^{1^{*}} & s_{1}^{1^{*}}+\tilde{s}_{3}^{1^{*}}-\hat{s}_{5}^{1^{*}}-\check{s}_{7}^{1^{*}} \\
s_{1}^{1}-\tilde{s}_{3}^{1}-\hat{s}_{5}^{1}+\check{s}_{7}^{1} & s_{2}^{1}-\tilde{s}_{4}^{1}-\hat{s}_{6}^{1}+\check{s}_{8}^{1} \\
-s_{2}^{1^{*}}+\tilde{s}_{4}^{1^{*}}+\hat{s}_{6}^{1^{*}}-\check{s}_{8}^{1^{*}} & s_{1}^{1^{*}}-\tilde{s}_{3}^{1^{*}}-\hat{s}_{5}^{1^{*}}+\check{s}_{7}^{1^{*}} \\
\vdots & \vdots \\
s_{1}^{M}+\tilde{s}_{3}^{M}-\hat{s}_{5}^{M}-\check{s}_{7}^{M} & s_{2}^{M}+\tilde{s}_{4}^{M}-\hat{s}_{6}^{M}-\check{s}_{8}^{M} \\
-s_{2}^{M^{*}}-\tilde{s}_{4}^{M^{*}}+\hat{s}_{6}^{M^{*}}+\check{s}_{8}^{M^{*}} & s_{1}^{M^{*}}+\tilde{s}_{3}^{M^{*}}-\hat{s}_{5}^{M^{*}}-\check{s}_{7}^{M^{*}} \\
s_{1}^{M}-\tilde{s}_{3}^{M}-\hat{s}_{5}^{M}+\check{s}_{7}^{M} & s_{2}^{M}-\tilde{s}_{4}^{M}-\hat{s}_{6}^{M}+\check{s}_{8}^{M} \\
-s_{2}^{M^{*}}+\tilde{s}_{4}^{M^{*}}+\hat{s}_{6}^{M^{*}}-\check{s}_{8}^{M^{*}} & s_{1}^{M^{*}}-\tilde{s}_{3}^{M^{*}}-\hat{s}_{5}^{M^{*}}+\check{s}_{7}^{M^{*}}
\end{array}\right],
$$

Note that the code provides one symbol per OFDM per radiation.

\section{RESULTS}

The simulation results will present in this section. In the proposed work, the author assumed that he have these proporties: $\mathrm{P}=2, \mathrm{M}_{\mathrm{t}}=2, \mathrm{M}_{\mathrm{r}}=1$ and $\mathrm{N}_{\mathrm{c}}=128$.

In his simulations, he assumed that the channel is perfect, and he chose a BPSK constellation, $1 \mathrm{bit} / \mathrm{sec} / \mathrm{Hz}$ spectral efficiency, where ignored the cyclic prefix (overhead). Fig. 2 shows the results at delay spread of $\tau=5 \mu \mathrm{s}$.

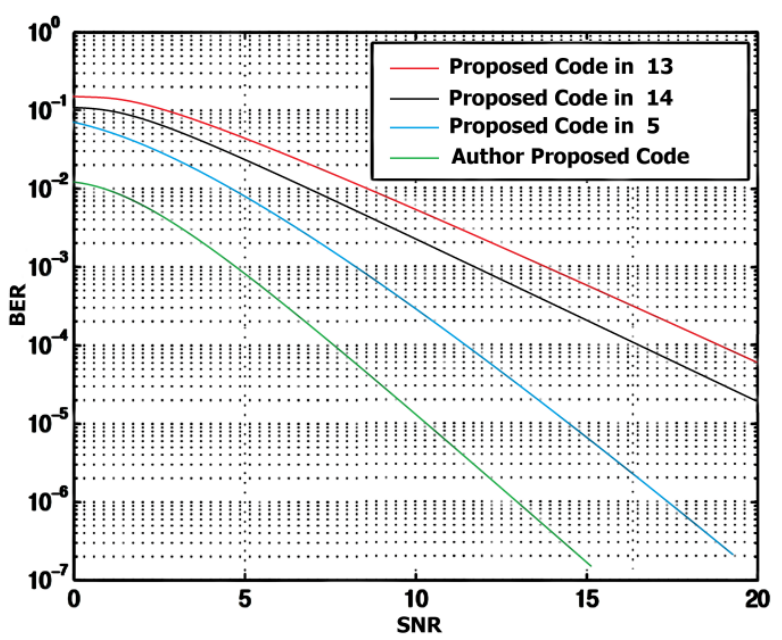

Fig. 2. BER vs SNR for the Proposed System.

The performance improving compared to [13], [14] and [5] is shown in the figure above.

\section{CONCLUSION}

The author implemented a NOVEL MIMO - OFDM technique for improving the wireless communications systems performance based on $\mathrm{SF}-\mathrm{BC}$.

Where, the designed code is based on the principle of QOC using the techniques of the reconfigurable antennas.

And he provided simulation results using MATLAB program to prove the NOVELTY of his code with comparison with the other SF codes.

Where the implemented code produces additional higher coding gains and diversity (Low BER at Low SNR) compared with other technique.

\section{ACKNOWLEDGMENT}

The author would like to thank THE GOD, the almighty, who gave him the reconcile to complete his research. Only due to THE GOD blessing, he could finish it successfully.

\section{REFERENCES}

[1] Y.Wu, and W.Y.Zou, "Orthogonal frequency division multiplexing a multi-carrier modulation scheme," IEEE Trans. on Consumer Electronics, vol. 41, no. 3, pp. 392399, Aug. 1995.

[2] Yang, Z. Bai, W. Liu, Z., "A Decision-Aided Residual ISI Cancellation Algorithm for OFDM Systems," Signal Processing, 2006 8th IEEE International Conference on, vol.3, pp.16-20, 2006.

[3] R.j Baxley, and T.Zhou, "PoIr Saving Analysis of Peakto-Average PoIr Ratio Reduction in OFDM". IEEE Transactions on Consumer Electronics, VOL.50, NO.3,pp.792-798, August 2004 World Academy of Science, Engineering and Technology 502009.

[4] Costantine J., Tawk Y., Barbin S.E., Christodoulou C.G. "Reconfigurable Antennas: Design and Applications", IEEE, pp. 424 - 437, March 2015.

[5] Vida Vakilian, Jean-Francois Frigon and Sandip Roy "Space-frequency block code for MIMO-OFDM communication systems with reconfigurable antennas", IEEE Wireless Communications, pp. 4221-4225, 2013.

[6] A. Grau, H. Jafarkhani, and F. De Flaviis, "A reconfigurable multipleinput multiple-output communication system," IEEE Trans. on Wireless Commun., vol. 7, pp. 1719-1733, May 2008.

[7] F. Fazel, A. Grau, H. Jafarkhani, and F. Flaviis, "Spacetime-state block coded MIMO communication systems using reconfigurable antennas," IEEE Trans. on Wireless Commun., vol. 8, pp. 6019-6029, Dec. 2009.

[8] D. Agrawal, V. Tarokh, A. Naguib, and N. Seshadri, "Space-time coded OFDM for high data-rate wireless communication over wideband channels," in Proc. IEEE Veh. Technol. Conf. (VTC), vol. 3, 1998, pp. 22322236.

[9] K. F. Lee and D. B. Williams, "A space-time coded transmitter diversity technique for frequency selective fading channels," in in Proc. IEEE Sensor Array and Multichannel Signal Processing Workshop, March 2000, pp. 149-152.

[10] K. F. Lee and D. B. Williams, "A space-frequency transmitter diversity technique for OFDM systems," in 
Proc. IEEE Global Commun. Conf. (GLOBECOM), vol. 3, 2000, pp. 1473-1477.

[11] A. F. Molisch, M. Z. Win, and J. H. Winters, "Spacetime-frequency (STF) coding for MIMO-OFDM systems," IEEE Commun. Lett., vol. 6, pp. 370-372, Sept. 2002.

[12] W. Su, Z. Safar, M. Olfat, and K. R. Liu, "Obtaining fulldiversity spacefrequency codes from space-time codes via mapping," IEEE Trans. Signal Process., vol. 51, no. 11, pp. 2905-2916, Nov. 2003.

[13] W. Su, Z. Safar, and K. R. Liu, "Full-rate full-diversity space-frequency codes with optimum coding advantage," IEEE Trans. Inf. Theory, vol. 51, pp. 229-249, Jan. 2005.

[14] F. Fazel and H. Jafarkhani, "Quasi-orthogonal spacefrequency and space-time-frequency block codes for
MIMO OFDM channels," IEEE Trans. on Wireless Commun., vol. 7, pp. 184-192, Jan. 2008.

[15] O. Tirkkonen, “Optimizing space-time block codes by constellation rotations," in Finnish Wireless Commun. Workshop (FWWC), Oct. 2001, pp. 59-60.

[16] W. Su and X.-G. Xia, "Signal constellations for quasiorthogonal spacetime block codes with full diversity," IEEE Trans. Inf. Theory, vol. 50, pp. 2331-2347, Oct. 2004.

\section{AUTHOR PROFILE}

Ali Jasim Ramadhan (Member IEEE) had B.Sc. degree in electrical engg., univ. of kufa and he has M.Sc. degree in electronics and communications engg., univ. of babylon, and now he is lecturer in computer engg. tech., univ. college of humanity studies and he holds a Patent (international classification) and he is member in Iraqi \& Arab engineers union. 